\title{
Princípios de design de uma experiência baseada no TPACK na formação inicial de professores de Matemática
}

\author{
Design principles of an TPACK-based experience in the Mathematics Pre-service \\ Teacher Education
}

\author{
Luis Fabián Gutiérrez-Fallas ${ }^{1}$ \\ Ana Henriques ${ }^{2}$
}

\begin{abstract}
Resumo
O TPACK (Technological Pedagogical Content Knowledge) conceptualiza o conhecimento profissional do professor para uma efetiva integração da tecnologia na educação, que é um desafio frequente no ensino da Matemática. Sendo essencial garantir o seu desenvolvimento na formação inicial de professores de Matemática, este estudo, seguindo uma Investigação Baseada em Design, visa compreender como os princípios de design adotados numa experiência de formação centrada no desenvolvimento do TPACK de futuros professores (FP) de Matemática do $7 .^{\circ}$ ao $12 .^{\circ}$ anos de escolaridade contribuem para promover esse conhecimento. Os resultados, com base na análise de resoluções escritas dos FP das tarefas propostas na formação e respostas a entrevista e questionário, mostram que a experiência de formação promoveu competências tecnológicas dos FP, evidenciando a mobilização do TK (Technological Knowledge), TCK (Technological Content Knowledge) e TPK (Technological Pedagogical Knowledge) em articulação com o PCK (Pedagogical Content Knowledge) e, consequentemente, com o TPACK.
\end{abstract}

Palavras-chave: TPACK; Formação inicial de professores de Matemática; Investigação Baseada em Design.

\begin{abstract}
TPACK (Technological Pedagogical Content Knowledge) conceptualizes the teacher's professional knowledge for an effective integration of technology in education, which is often a challenge in mathematics teaching. Since it is essential to guarantee its development in pre-service mathematics teacher education, this study aims, following a Design-Based Research, to understand how the design principles adopted in a teacher education experience centred on developing the TPACK of mathematics prospective teachers (PTs) of 7th to 12th school years contribute to promote this knowledge. The results, based on the analysis of PTs' written solutions of the tasks proposed in the teacher training and responses to an interview and questionnaire, show that the teacher education experience promoted PTs' technological skills, evidencing the mobilization of TK (Technological Knowledge), TCK (Technological Content Knowledge) and TPK (Technological Pedagogical Knowledge) in articulation with the PCK (Pedagogical Content Knowledge) and, consequently, the TPACK.
\end{abstract}

Keywords: TPACK; Pre-service mathematics teacher education; Design-Based Research

\footnotetext{
Submetido em: 29/10/2020 - Aceito em: 04/03/2021 - Publicado em: 27/05/2021

${ }^{1}$ Doutor em Educação, especialidade Didática da Matemática pela Universidade de Lisboa, Portugal. Professor do Departamento de Educação Matemática da Universidade da Costa Rica, Costa Rica. Email: professor.fgutierrez@gmail.com. ORCID: https://orcid.org/0000-0001-9089-2062.

${ }^{2}$ Doutora em Educação, especialidade Didática da Matemática pela Universidade de Lisboa, Portugal. Professora do Instituto de Educação da Universidade de Lisboa, Portugal. Email: achenriques@ie.ulisboa.pt. ORCID: https://orcid.org/0000-0001-7844-2157.
} 


\section{Introdução}

A evolução das tecnologias digitais no século XXI e a sua ampla acessibilidade e potencialidade para fins educacionais levanta diversos questionamentos sobre $\mathrm{o}$ conhecimento profissional do professor para responder aos desafios e exigências de uma educação significativamente transformada pelo seu uso (Albuquerque et al., 2006; Mishra \& Koehler, 2006; Niess, 2012a). O modelo TPACK (Technological Pedagogical and Content Knowledge), proposto por Mishra e Koehler (2006), tem-se consolidado na conceptualização do conhecimento que os professores necessitam para uma efetiva integração da tecnologia digital no ensino, influenciando recentes investigações sobre o desenvolvimento desse conhecimento (Gutiérrez-Fallas \& Henriques, 2020; Niess \& Gillow-Wiles, 2017).

$\mathrm{Na}$ Educação Matemática, em particular, as orientações curriculares recomendam o uso da tecnologia como recurso essencial na aprendizagem dos alunos, apoiando-os a atribuir significado às ideias matemáticas, a raciocinar e a comunicar o seu pensamento (NCTM, 2014). Para responder a estas exigências, que se têm revelado um desafio para os professores, é fundamental que os programas de formação inicial "integrem diversas tecnologias educacionais que estejam acessíveis aos formandos" (Gutiérrez-Fallas \& Henriques, 2020. p. 200), para os envolverem na sua exploração, proporcionando oportunidades aos futuros professores (FP) de Matemática de desenvolverem o seu TPACK para criarem situações de aprendizagem, integrando as tecnologias, que apoiem a aprendizagem da Matemática dos alunos (AMTE, 2017; Niess, 2012a). No entanto, apesar do esforço que tem sido feito para integrar a tecnologia na formação inicial de professores, diversos estudos (Niess, 2012a; Niess \& Gillow-Wiles, 2017) revelam dificuldades nessa integração ainda pouco considerada , pelo que a preparação de FP para ensinar Matemática com tecnologia é uma área que requer mais investigação (Niess, 2012b), nomeadamente investigações focadas em princípios de design a considerar em contextos específicos de formação inicial de professores para promover o TPACK.

Neste contexto, foi pertinente realizar um estudo com base numa inovadora experiência de formação com FP de Matemática, seguindo uma metodologia de investigação baseada em design (Cobb, Confrey, diSessa, Lehrer \& Schauble, 2003), que visa desenvolver uma teoria local sobre como desenvolver o seu TPACK no contexto da formação inicial.

Visando contribuir com um modo inovador e específico de desenvolver o TPACK em contexto de formação inicial, que pode ser útil para a comunidade científica e educativa, neste artigo apresentamos um estudo com o objetivo de compreender como os princípios de design adotados numa experiência de formação, centrada no desenvolvimento do TPACK de futuros professores (FP) de Matemática do $7 .^{\circ}$ ao $12 .^{\circ}$ anos de escolaridade, contribuem para promover esse conhecimento. 


\section{O TPACK na formação inicial de professores de Matemática}

Os professores de Matemática têm sido encorajados a usar tecnologia nas suas práticas pedagógicas, particularmente a tecnologia educacional, por ser um recurso que influencia a Matemática que é ensinada e o modo como os alunos aprendem, sendo reconhecido o seu potencial para melhorar esses processos (NCTM, 2014; AMTE, 2017). No entanto, como Earle (2002) argumenta, a integração da tecnologia no ensino não deve ter como foco a própria tecnologia, mas aimplementação de práticas em que os alunos se envolvam ativamente tornando a sua aprendizagem mais significativa.

Para Krumsvik (2014) "há uma necessidade de desenvolver tanto fundamentos como modelos teóricos para uma compreensão mais profunda da competência digital na formação de professores" (p. 272). Nesse sentido, algumas organizações internacionais têm proposto modelos que visam definir princípios ou normas sobre o uso da tecnologia pelos professores e orientam a sua integração nos programas de formação inicial de professores. Por exemplo, a UNESCO (2008) apresenta um conjunto de normas orientadoras do uso da tecnologia na formação inicial de professores num framework (UNESCO ICT Competency Framework for Teachers, ICT-CFT), no qual define os seguintes três níveis das competências dos professores no uso e na integração da tecnologia:

1. Literacia tecnológica. Neste primeiro nível espera-se que as competências dos professores incluam habilidades básicas de alfabetização digital e cidadania digital, juntamente com a capacidade de selecionar e usar tutoriais, jogos, software e a web. Também devem usar as tecnologias para gerenciar dados de sala de aula e apoiar a sua própria aprendizagem profissional.

2. Aprofundamento do conhecimento. No segundo nível as competências do professor incluem a capacidade de gerenciar informações, estruturar tarefas problemáticas e integrar ferramentas, como softwares, no ensino e na aprendizagem de conteúdos específicos mediante estratégias de ensino centradas no aluno. Também devem apoiar projetos colaborativos, para o qual devem usar recursos em rede e baseados na web, para criar e monitorar planos de projetos dos alunos, bem como para colaborar com outros professores e para apoiar a sua própria aprendizagem profissional.

3. Criação do conhecimento. No terceiro nível espera-se que os professores competentes elaborem ambientes de aprendizagem suportados em ferramentas tecnológicas, e que as usem para apoiar o desenvolvimento de conhecimento e da capacidade de pensamento crítico dos alunos, a sua aprendizagem contínua e reflexiva, e criar comunidades de conhecimento para alunos e colegas. Também serão capazes de desempenhar um papel de liderança com os colegas na criação e implementação de uma visão da escola como uma comunidade baseada na inovação e na aprendizagem contínua, enriquecida pela tecnologia.

Esta ênfase na integração da tecnologia na Educação fez emergir a necessidade de desenvolver modelos para representar o conhecimento do professor necessário para efetivar 
DOI: $10.20396 /$ zet.v29i00.8661780

com sucesso essa integração. Mishra e Koehler (2006) propõem um modelo teórico, o TPACK, caraterizado pela integração simultânea e relacional de três domínios do conhecimento profissional do professor: conteúdo, pedagogia e tecnologia (Figura 1). Esta integração resulta no surgimento de sete tipos de conhecimento que conformam o framework do TPACK (Mishra \& Koehler, 2006): conhecimento do conteúdo (CK), conhecimento pedagógico (PK), conhecimento tecnológico (TK), conhecimento pedagógico do conteúdo (PCK), conhecimento tecnológico e pedagógico (TPK), conhecimento tecnológico do conteúdo (TCK), e conhecimento tecnológico e pedagógico do conteúdo (TPACK). Em particular, consideramos os conhecimentos CK, PK e PCK como conhecimentos clássicos que têm sido discutidos por distintos autores desde os anos 80 (Grossman, 1989; Shulman, 1986), e consideramos o TPK, o TCK e o TPACK como conhecimentos emergentes, uma vez que emergem ao integrar o TK com o CK e o PK.

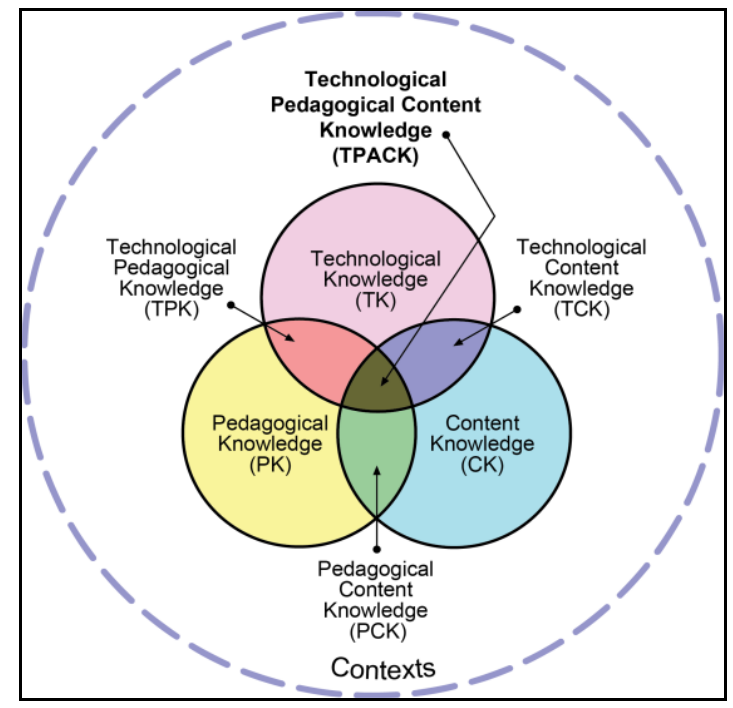

Figura 1. Technological Pedagogical Content Knowledge Model.

Fonte: Koehler et al. (2014, p. 103). Reproduzido com permissão do editor, (C) 2012 disponível em tpack.org

O modelo TPACK sugere que os professores precisam de ter uma profunda compreensão de cada um dos domínios deste conhecimento para planificar e desenvolver atividades curriculares que visam orientar e promover nos alunos uma aprendizagem com tecnologia. No entanto, o TPACK é mais do que conhecimento de conteúdo, pedagogia e tecnologia, considerados individualmente, mas envolve uma relação dinâmica entre estes domínios do conhecimento e as habilidades do professor para ensinar conteúdos específicos em níveis escolares também específicos (Koehler, Mishra, Kereluik, Shin \& Graham, 2014; Niess, 2012b).

Segundo Ponte (2012), planificar, desenvolver o currículo, promover a aprendizagem e ensinar conteúdos específicos, constituem ações do professor associadas ao seu conhecimento didático. Para este autor: 
DOI: $10.20396 /$ zet.v29i00.8661780

O conhecimento profissional do professor de Matemática inclui diversos aspetos, dos quais nos interessa sobretudo o que se refere à prática letiva, aquele onde se faz sentir de modo mais forte a especificidade da disciplina de Matemática, e que designamos por conhecimento didático (Ponte, 2012, pp. 86-87).

Nesta perspectiva, o TPACK assume-se como um conhecimento profissional especializado, e em particular neste estudo, como o conhecimento didático do professor necessário para integrar efetivamente a tecnologia no ensino e na aprendizagem da Matemática.

A formação inicial de professores de Matemática é um processo complexo e influenciado por vários elementos que interagem entre si (Ponte \& Chapman, 2008). Um professor de Matemática deverá ter conhecimento sobre: a natureza da Matemática; os conteúdos matemáticos; os objetivos curriculares; o modo como os alunos compreendem e aprendem os conteúdos matemáticos; a forma de apresentar as ideias aos alunos para que sejam aprendidas; e a gestão da sala de aula (Albuquerque et al., 2006). Esta complexidade do conhecimento profissional do professor levanta questões sobre o processo de formação dos FP, nomeadamente como prepará-los para enfrentarem os desafios com que se irão confrontar na futura prática profissional.

Parte destes desafios emergem do constante desenvolvimento tecnológico, sobretudo o software educacional, e das recomendações curriculares sobre o seu uso (NCTM, 2014). Será então necessário que a formação inicial de professores promova o desenvolvimento de um conhecimento aprofundado relativo à integração da tecnologia no ensino e na aprendizagem da Matemática, cumprindo as recomendações da AMTE (2017) sobre o perfil do futuro professor de Matemática que o caracteriza como proficiente em ferramentas tecnológicas, quer para seu próprio na Matemática como para apoiar a aprendizagem matemática dos alunos.

A este respeito, Niess (2012a) sugere que os programas de formação sejam planeados para preparar os FP para o ensino da Matemática com tecnologia, sendo esta aprendizagem um processo de aquisição de conhecimento tecnológico e de articulação com o conhecimento didático, considerando como as tecnologias podem ter impacto nas estratégias de ensino, no currículo escolar e no modo como os alunos aprendem os conteúdos. Na literatura têm surgido várias abordagens para desenvolver o TPACK dos FP que, segundo Koehler et al. (2014), podem classificar-se em três possíveis caminhos (Figura 2): (i) do PCK ao TPACK, (ii) do TPK ao TPACK, e (iii) PCK e TPACK em simultâneo. Os autores salientam ainda que estes caminhos não são necessariamente disjuntos, pelo contrário, existe alguma superposição entre estas diferentes abordagens. 
DOI: $10.20396 /$ zet.v29i00.8661780

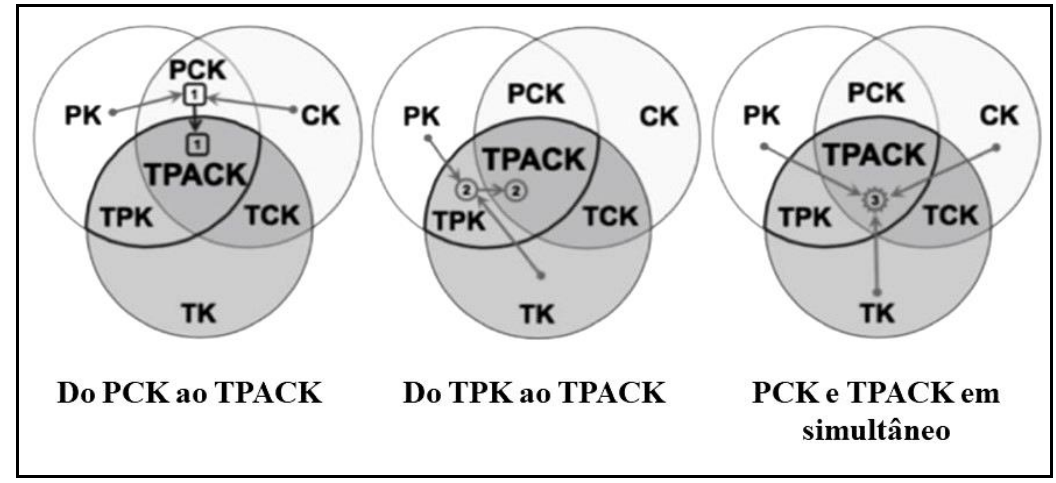

Figura 2. Caminhos para desenvolver o TPACK

Fonte: Koehler et al. (2014, p. 106)

Do PCK ao TPACK. Nesta abordagem, a tecnologia é introduzida como uma forma de apoiar e aprofundar as estratégias de ensino já estabelecidas e utilizadas pelo professor. Isto é, primeiro o professor desenvolve o PCK através de experiências que não envolvem o uso de tecnologia e mais tarde, o professor aprende como a tecnologia pode ser usada para aperfeiçoar as estratégias com as quais já está familiarizado. Os autores salientam que uma das limitações deste caminho está conformada pelo conjunto de conceções e crenças que o futuro professor tem interiorizado sobre o ensino e aprendizagem de determinado conteúdo, o que limita a sua visão e disposição para experimentar novas estratégias apoiadas pela tecnologia.

Do TPK ao TPACK. Esta abordagem usa-se quando um futuro professor participa numa disciplina de formação sobre tecnologia antes de desenvolver o PCK, que visa abordar amplamente a tecnologia em diferentes áreas, como o uso da Web 2.0, software e outras ferramentas digitais, mas não conhece ainda estratégias pedagógicas específicas para o ensino dos conteúdos. Deste modo, o primeiro passo neste caminho é desenvolver o TK e o TPK nas experiências iniciais, depois passando pelas disciplinas pedagógicas, o futuro professor desenvolve o PCK e expande o TPK em TPACK. Finalmente, os autores salientam que esta "é a "abordagem padrão" na maioria das instituições de ensino superior. A tecnologia é relegada para algumas disciplinas, e os professores são encaminhados para frequentar essas aulas e aplicar o conhecimento adquirido às suas próprias áreas de conteúdo" (p. 107, aspas no original).

PCK e TPACK em simultâneo. Num contexto de formação inicial de professores, este caminho consiste em integrar sistematicamente a tecnologia nas disciplinas de metodologia, pedagogia ou didática na área específica do conteúdo. Isto é, um programa de formação de professores que segue esta abordagem, pode não ter uma disciplina específica de tecnologia, mas promover o uso da tecnologia para ensinar conteúdos nas diferentes disciplinas de metodologia e didática da sua respetiva área profissional. Assim os FP desenvolverão o seu PCK e o seu TPACK simultaneamente. Segundo os autores, um dos desafios que se levanta nesta abordagem é a carga cognitiva à qual os FP estão expostos quando tentam desenvolver simultaneamente conhecimentos associados aos domínios 
pedagógico, do conteúdo e tecnológico.

No estudo apresentado neste artigo, a experiência de formação segue o terceiro caminho, PCK e TPACK em simultâneo, assumindo que esta abordagem promove oportunidades para os FP integrarem a tecnologia em articulação com a Matemática e a sua Didática nas tarefas e atividades propostas na disciplina de didática da Matemática. E também se pretende que "os professores experimentem por si mesmos, enquanto aprendizes, as potencialidades e limitações de ferramentas digitais na aprendizagem da Matemática, para assim adquirirem conhecimento sobre como os alunos podem aprender Matemática em vários ambientes digitais" (Leung, 2017, p. 6).

\section{Metodologia do estudo}

\section{Opções metodológicas, contexto e participantes}

Este estudo, apoiado numa experiência de formação inicial (Gutiérrez-Fallas, 2019), segue uma metodologia de Investigação Baseada em Design (IBD), contempando dois ciclos de design completos (preparação, experimentação e análise retrospetiva) (Cobb et al., 2003; Ponte, Carvalho, Mata-Pereira \& Quaresma, 2016), para desenvolver uma teoria local sobre como promover o TPACK de FP de Matemática.

A fase de preparação consistiu na formulação dos objetivos formativos da experiência para desenvolver o TPACK, na planificação de tarefas e na formulação dos princípios de design subjacentes à intervenção a realizar. A fase de experimentação contemplou a implementação das tarefas em 11 sessões da formação ( $2 \mathrm{~h}$ cada), seguindo uma abordagem de ensino exploratório (Canavarro, 2011) que contempla três momentos: (i) apresentação e introdução da tarefa, dando instruções para a resolução da tarefa; (ii) resolução autónoma da tarefa pelos FP, trabalhando individualmente ou em pares; e (iii) discussão coletiva, que consistiu num espaço de reflexão e partilha das resoluções das tarefas. Finamente, a fase de análise retrospectiva teve o propósito de redefinir os princípios de design com base na reflexão sobre os resultados obtidos na experimentação da proposta.

Em ambos os ciclos de design, a experiência de formação focada no desenvolvimento do TPACK de FP de Matemática de níveis de escolaridade do $7 .^{\circ}$ ao $12 .^{\circ}$ ano, foi realizada numa disciplina de Didática do 2. ${ }^{\circ}$ semestre do 1. ${ }^{\circ}$ ano do curso de Mestrado em Ensino de Matemática de uma universidade portuguesa que proporciona habilitação profissional para a docência. Esta disciplina visa proporcionar aos FP os instrumentos didáticos fundamentais para o ensino da Matemática, abordando temáticas associadas à aprendizagem do tema, à gestão curricular, à avaliação das aprendizagens e aos recursos didáticos, incluindo a tecnologia. Os autores desde artigo tiveram o papel de formadores, sendo a segunda autora a professora titular da disciplina e o primeiro autor um professor colaborador.

Os FP que frequentavam a disciplina de Didática em cada ciclo de design em que decorreu a experiência de formação, participaram neste estudo de forma voluntária através de um consentimento informado entregue na primeira semana do semestre letivo. No $1 .^{\circ}$ ciclo 
participaram 6 futuras professoras (Patrícia, Cristina, Sara, Vitória, Marta e Paula) e, no 2. ${ }^{\circ}$ ciclo, 4 futuras professoras e 2 futuros professoras (Ana, Sofia, Glória, Isabel, Samuel e Tiago), cujos nomes são fictícios. Estes FP, licenciados em Matemática, adquiriram algum conhecimento pedagógico numa disciplina prévia da formação inicial, mas sem envolver tecnologia. Só uma futura professora indicou ter tido formação em tecnologia em cursos extracurriculares, os restantes indicaram que não tiveram formação prévia complementar na área de tecnologia em geral ou em educação. No entanto, assumem ter algum conhecimento tecnológico adquirido autonomamente através da experiência com as tecnologias do seu quotidiano.

A análise dos dados recolhidos na fase de experimentação da experiência de formação inicial, em ambos os ciclos da IBD, foi feita de forma descritiva e interpretativa (Coutinho, 2011) com foco no design da experiência baseada no TPACK e respetivo referencial teórico, descrito a seguir. Neste estudo foram analisadas: as resoluções escritas (RT\#) dos FP de tarefas propostas nas sessões de formação; os registos áudio e vídeo da entrevista semiestruturada (E) e as respostas ao questionário aberto $(\mathrm{Q})$, ambos realizados com os FP no final de cada fase de experimentação e focados no conhecimento adquirido na experiencia de formação, sendo solicitados a reportar: as suas atitudes, utilização e conhecimento sobre a tecnologia; e conceções sobre o contributo do trabalho realizado para o seu conhecimento profissional e integração da tecnologia na prática futura.

Estes dados foram selecionados por ilustrarem conhecimentos relativos a TPACK adquiridos pelos FP, e cuja análise se pretende que seja representativa da experiência de formação inicial realizada e de como pode contribuir para desenvolver o TPACK emquanto conhecimento profissional dos FP necessário para integrar efetivamente a tecnologia no ensino e na aprendizagem da Matemática.

\section{A experiência de formação baseada no TPACK: características e princípios de design}

A experiência de formação está enquadrada no estudo de Gutiérrez-Fallas (2019), dentro do programa da disciplina de Didática e suportada no framework do TPACK (Mishra \& Koehler, 2006), considerando que o seu objetivo principal é desenvolver o TPACK de FP de Matemática. Em particular, os objetivos que se procuraram desenvolver na formação inicial são:

- Aprimorar as conceções dos FP sobre a integração da tecnologia no ensino e na aprendizagem da Matemática.

- Articular o conhecimento tecnológico com o conhecimento didático dos FP para o uso eficiente das ferramentas tecnológicas no ensino e na aprendizagem da Matemática.

Para atingir estes objetivos, a experiência baseada no TPACK contemplou duas dimensões principais. A dimensão designada por prática-pedagógica consistiu na definição de uma abordagem que orienta a experimentação da experiência de formação na sala de aula. Esta abordagem segue uma Trajetória de Formação e Aprendizagem (Figura 3) que envolveu doze tarefas de natureza aberta que estimulam a inquirição autónoma e protagonismo dos FP 
DOI: $10.20396 /$ zet.v29i00.8661780

na sua realização e momentos de partilha e discussão coletiva de processos e respostas (Ponte \& Chapman, 2008), e contemplou três fases: (i) experiências iniciais, (ii) experiências de formação e aprendizagem, e (iii) experiências de produção.

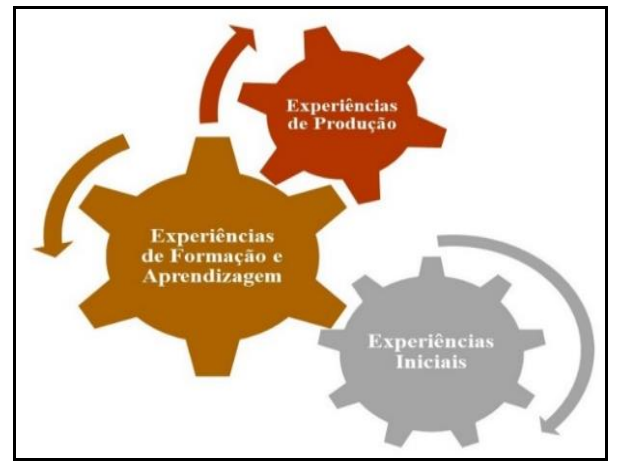

Figura 3. Trajetória de Formação e Aprendizagem da experiência baseada no TPACK

Na fase 1 pretende-se explorar as conceções que os FP têm sobre a integração da tecnologia na Educação Matemática e promover a articulação dessas conceções com o seu conhecimento didático. A fase 2 tem como objetivo desenvolver o conhecimento tecnológico dos FP através da exploração de diferentes ferramentas tecnológicas para o ensino da Matemática. Finalmente, na fase 3, procura-se promover a mobilização e operacionalização do TPACK dos FP na elaboração de propostas didáticas envolvendo tarefas, planos de aula e momentos de reflexão e partilha de conhecimentos. Na Tabela 1 apresenta-se uma descrição geral das tarefas realizadas pelos FP em cada uma destas fases.

Tabela 1 - Descrição geral das tarefas da experiência baseada no TPACK (Gutiérrez-Fallas, 2019)

\begin{tabular}{|c|c|c|}
\hline Fase & Tarefa & Descrição \\
\hline \multirow{6}{*}{$\begin{array}{l}\text { Fase } 1 . \\
\text { Experiências } \\
\text { iniciais }\end{array}$} & T1. Participação num fórum & Discussão sobre a integração da tecnologia na \\
\hline & da plataforma moodle & Educação Matemática, com base na leitura de texto \\
\hline & T2. Explorando recursos & Reconhecer diversas ferramentas tecnológicas \\
\hline & tecnológicos & $\begin{array}{l}\text { disponíveis e gratuitas em internet para o ensino e } \\
\text { aprendizagem da Matemática }\end{array}$ \\
\hline & $\begin{array}{l}\text { T3. Análise de tarefa } \\
\text { matemática }\end{array}$ & $\begin{array}{l}\text { Análise reflexiva sobre a integração da tecnologia } \\
\text { numa tarefa matemática }\end{array}$ \\
\hline & $\begin{array}{l}\text { T4. Análise de plano de } \\
\text { aula }\end{array}$ & $\begin{array}{l}\text { Análise reflexiva da proposta de integração da } \\
\text { tecnologia num plano de aula disponibilizado }\end{array}$ \\
\hline \multirow{4}{*}{$\begin{array}{l}\text { Fase } 2 . \\
\text { Experiências } \\
\text { de formação e } \\
\text { aprendizagem }\end{array}$} & $\begin{array}{l}\text { T5. Explorando o } \\
\text { GeoGebra }\end{array}$ & $\begin{array}{l}\text { Uso do GeoGebra na resolução de uma tarefa de } \\
\text { geometria plana pelos FP }\end{array}$ \\
\hline & $\begin{array}{l}\text { T6. Explorando a } \\
\text { calculadora }\end{array}$ & $\begin{array}{l}\text { Análise do uso da calculadora gráfica por alunos na } \\
\text { resolução de uma tarefa envolvendo funções }\end{array}$ \\
\hline & T7. Explorando applets & $\begin{array}{l}\text { Pesquisa, exploração e análise reflexiva de uma } \\
\text { applet disponível num website para o ensino e } \\
\text { aprendizagem de algum conteúdo matemático }\end{array}$ \\
\hline & $\begin{array}{l}\text { T8. Explorando o } \\
\text { TinkerPlots }\end{array}$ & $\begin{array}{l}\text { Uso do TinkerPlots } \\
\text { estatística pelos } \text { FP }\end{array}$ \\
\hline \multirow{3}{*}{$\begin{array}{l}\text { Fase } 3 . \\
\text { Experiências } \\
\text { de produção }\end{array}$} & $\begin{array}{l}\text { T9. Elaboração de uma } \\
\text { tarefa matemática }\end{array}$ & $\begin{array}{l}\text { Seleção e adaptação de uma tarefa matemática que } \\
\text { integre o uso da tecnologia na sua resolução }\end{array}$ \\
\hline & $\begin{array}{l}\text { T10. Elaboração de um } \\
\text { plano de aula }\end{array}$ & $\begin{array}{l}\text { Proposta didática para uma aula de Matemática, } \\
\text { com base numa tarefa que integre o uso de } \\
\text { ferramenta tecnológica explorada na disciplina }\end{array}$ \\
\hline & T11. Reflexão final & Relatório escrito de natureza reflexiva sobre a \\
\hline
\end{tabular}


DOI: 10.20396/zet.v29i00.8661780

T12. Elaboração de um portfólio digital integração da tecnologia na Educação Matemática Elaboração de um portfólio digital na plataforma WIX.com como recurso para a avaliação das aprendizagens dos FP

Fonte: Elaboração própria.

A segunda dimensão referida como teórica-investigativa, envolveu a elaboração de um conjunto de princípios de design subjacentes à experiência (Figura 4), que foram alvo de refinamento na análise retrospectiva realizada após a fase de experimentação em cada um dos ciclos da IBD. Este processo de aperfeiçoamento é orientado pela formulação de uma conjetura, suscetível de teste, que estrutura o design da experiência (Ponte et al., 2016).

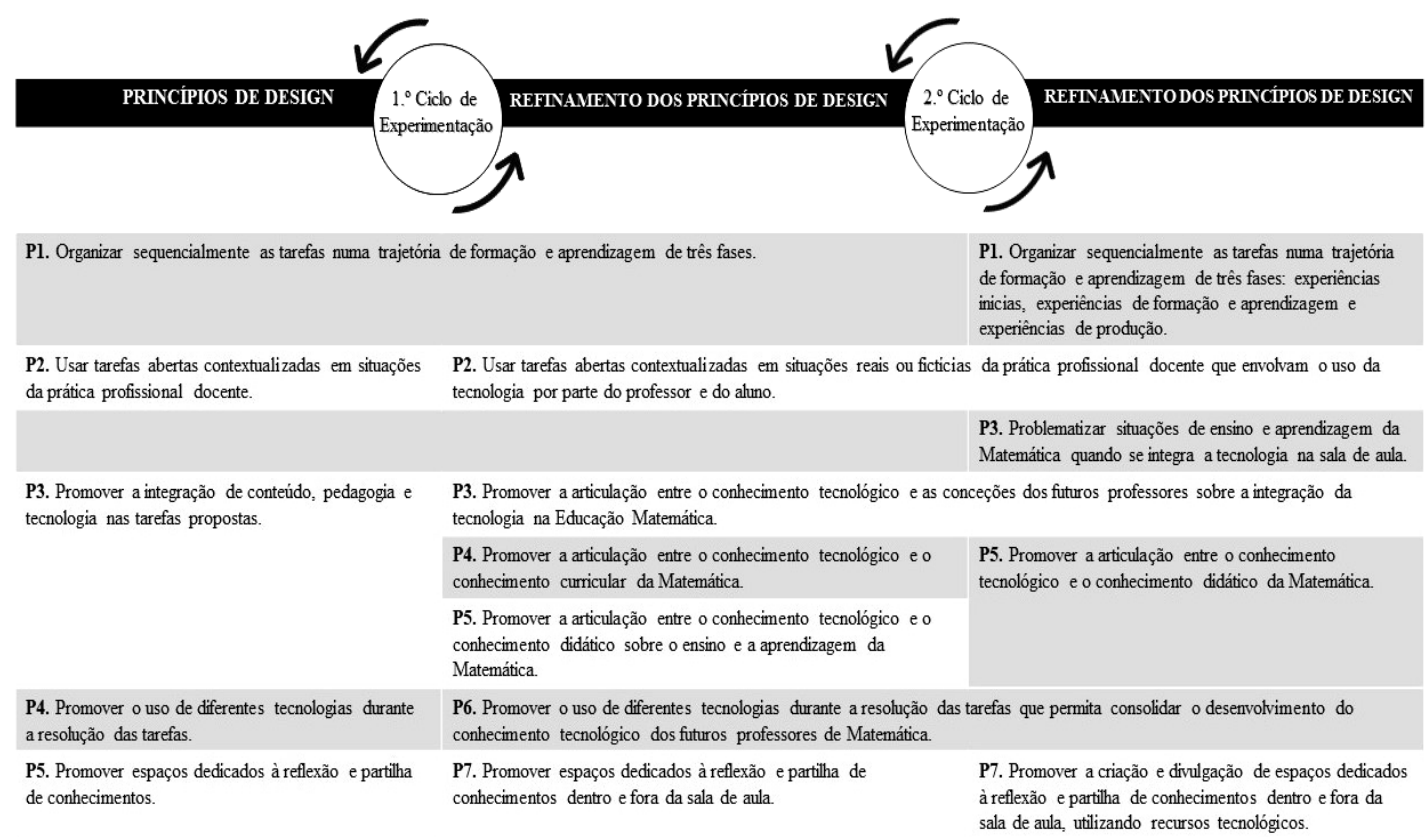

Figura 4. Refinamento dos princípios de design da experiência baseada no TPACK

Portanto, depois dos dois ciclos da IBD desenvolvida neste estudo, definem-se os seguintes sete princípios de design $(\mathrm{P} 1, \ldots, \mathrm{P} 7)$ que permitem apoiar uma experiência na formação inicial de professores de Matemática para desenvolver o TPACK:

P1. Organizar sequencialmente as tarefas numa trajetória de formação e aprendizagem de três fases: experiências iniciais, experiências de formação e aprendizagem e experiências de produção.

P2. Usar tarefas abertas contextualizadas em situações reais ou fictícias da prática profissional docente que envolvam o uso da tecnologia por parte do professor e do aluno.

P3. Problematizar situações de ensino e aprendizagem da Matemática quando se integra a tecnologia na sala de aula.

P4. Promover a articulação entre o conhecimento tecnológico e as conceções dos FP sobre a integração da tecnologia na Educação Matemática.

P5. Promover a articulação entre o conhecimento tecnológico e o conhecimento didático 
da Matemática.

P6. Promover o uso de diferentes tecnologias durante a resolução das tarefas que permita consolidar o desenvolvimento do conhecimento tecnológico dos FP de Matemática.

P7. Promover a criação e divulgação de espaços dedicados à reflexão e partilha de conhecimentos dentro e fora da sala de aula, utilizando recursos tecnológicos.

Assim, a conjetura testada e verificada durante os ciclos desta IBD, é que uma experiência de formação com as caraterísticas descritas e sustentada nestes princípios de design contribui para promover o TPACK dos FP de Matemática.

\section{Análise do design da experiência baseada no TPACK}

Nesta secção apresentamos os principais resultados da análise, evidenciada com excertos das resoluções escritas das tarefas dos FP e das sua respostas a entrevistas e questionário.

Em relação ao que a experiência de formação visava desenvolver, neste caso o TPACK como o conhecimento profissional dos FP necessário para integrar efetivamente a tecnologia no ensino e na aprendizagem da Matemática, os dados mostram que os FP reconheceram ter adquirido conhecimentos relativos à integração dos três domínios do conhecimento que constituem o TPACK: CK, PK e TK. Por exemplo, Ana assegura que esta experiência formativa "permitiu-me uma familiarização com todos estes elementos, (matemática, tecnologia e didática) o que potência a confiança do futuro professor" (RT11, negrito autor). Isabel também afirmou que "nas minhas práticas profissionais pretendo mobilizar os novos conhecimentos adquiridos, tanto na perspectiva dos conteúdos como na perspectiva da tecnologia" (RT5, negrito autor). Semelhantemente, Sara faz referência aos conhecimentos adquiridos e à visão de aplicá-los na sua futura prática profissional:

Todo o trabalho desenvolvido [na unidade curricular] permitiu-me adquirir conhecimentos importantes. Desta forma, me consciencializou que será importante, na minha futura prática profissional, que implemente as tecnologias da melhor forma, visando sempre aprendizagens e experiências bastante enriquecedoras nos alunos e no ensino da Matemática. (RT11, negrito autor)

Assim, as afirmações acima revelam que, para além da aquisição de conhecimento novo e importante, as FP também reconheceram a importância e a intenção de mobilizar estes conhecimentos na sua futura prática profissional. Glória também é um caso que destacou o contacto com tecnologias educativas durante esta experiência formativa como gerador do conhecimento dela sobre as ferramentas tecnológicas (TK), e sobre como as integrar didaticamente na sala de aula de Matemática para benefíciar a aprendizagem dos alunos (TPK), afirmando:

Me ajudou muito para conhecer novas tecnologias, usar a calculadora gráfica, que nunca tinha usado, software que não conhecia. Tudo vai ser de muita valia para o meu futuro, porque já conhecendo essas tecnologias já tenho uma ideia do que eu posso usar e como contribui para as aprendizagens dos alunos, já tenho uma primeira experiência, pois consegui nesta disciplina evitar um possível problema, conhecendo 
DOI: $10.20396 /$ zet.v29i00.8661780

uma nova ferramenta didática que muito provavelmente irei usar em sala de aula (RT11).

Relativamente ao como foi previsto desenvolver o TPACK dos FP, a análise da operacionalização dos princípios de design durante a experiência de formação, evidenciou a diversidade dos resultados seguintes.

No caso das tarefas de natureza aberta (P2), que buscavam desafiar os FP na articulação do TK com o PCK, este aspeto foi reconhecido quando, por exemplo na T9, Isabel afirma que "esta tarefa envolve pesquisa, escolha de tecnologia e adaptação da situação de aprendizagem, tornando-a mais desafiante" (RT9).

As tarefas abertas também se caracterizaram por promover a colaboração entre os FP, em cada um dos ciclos nove das tarefas foram resolvidas em pares, mantendo-se sempre os mesmos com o propósito de consolidar a afinidade entre os FP, assim como também fortalecer o seu desempenho e progresso no trabalho ao longo da experiência de formação. Os dados mostram que os FP destacaram aprendizagens geradas pelo trabalho colaborativo, como indicado por Vitória, "pelo facto de ter sido um trabalho de grupo, é importante destacar, também, como uma das minhas principais aprendizagens a experiência desenvolvida através do trabalho colaborativo" (RT11), e semelhantemente Paula afirma que “o trabalho em grupo é uma das aprendizagens que em minha opinião eu levo” (E).

A experiência baseada no TPACK procurou problematizar situações de ensino e aprendizagem da Matemática quando se integra a tecnologia (P3), através de tarefas que apelavam a contextos, hipotéticos ou reais, que descreviam situações de sala de aula que envolvia a integração da tecnologia. A este respeito, Paula indicou que em termos gerais durante a disciplina "foi feita uma abordagem à tecnologia e à forma como esta poderia ajudar os alunos no seu processo de ensino e aprendizagem" (RT11). Por sua vez, Glória especificou que:

Tivemos muitas tarefas relacionadas à tecnologia, tivemos muitas opiniões, muitas discussões sobre o uso da tecnologia no ensino, a tecnologia na sala de aula, o uso da tecnologia pelos professores, pelos alunos, como contribui a tecnologia para as aprendizagens dos alunos, quais são os riscos, as potencialidades, dificuldades, etc. (RT11).

Também se visou promover a articulação do conhecimento tecnológico com as conceções dos FP sobre a sua integração (P4). Os dados evidenciam o reconhecimento por parte dos FP da era digital que carateriza o século XXI e as implicações que isto tem na Educação Matemática. Por exemplo, Ana argumentou que "numa era tão tecnológica e cada vez mais digital como a que vivemos, abordar o tema da utilização da tecnologia como meio de promoção do processo ensino-aprendizagem, não só é útil como imprescindível para qualquer futuro professor" (RT2). Em termos do modo como as conceções sobre a importância de utilizar a tecnologia no ensino e na aprendizagem da Matemática foram consolidadas, Isabel argumenta que "eu sempre fui adepta da tecnologia, mas com a experiência nesta disciplina agarrei ainda mais argumentos para continuar a usar a tecnologia" (E). 
Os dados também permitiram identificar mudanças nas conceções de alguns FP. Por exemplo, Marta salienta que a sua perspectiva e posição sobre o uso de tecnologias em contextos educativos alterou-se após ter participado na experiência de formação, argumentando:

Primeiramente, no que diz respeito à utilização e importância da tecnologia no âmbito da educação, durante bastante tempo adotei uma posição contra a utilização de tecnologias em contexto de sala de aula, por considerar que seria um fator de distração muito elevado, afastando os alunos do foco principal da aula. No entanto, posteriormente à realização do trabalho aqui discutido, percebi que a tecnologia pode ser bastante útil, quando bem implementada, oferecendo ferramentas diversas para a exploração de certos contextos, o que, por vezes, se torna difícil de concretizar, em tempo útil, sem a utilização de recursos tecnológicos (RT11).

No que respeita a promover a articulação entre o conhecimento tecnológico e o conhecimento didático da Matemática (P5), os dados mostram que os FP mobilizaram o TK nas propostas didáticas elaboradas por eles (PCK), como a tarefa matemática ou os planos de aula. Por exemplo, Marta e Paula referem que "com o auxílio do software, os alunos poderão desenvolver algumas capacidades transversais, tais como o raciocínio indutivo, desenvolvimento da linguagem matemática, o trabalho autónomo e cooperativo" (RT9). Nos planos de aula, os FP também destacaram o envolvimento dos alunos numa exploração ativa de conceitos e ideias matemáticas (CK) com a ferramenta tecnológica (TK). Nomeadamente, no plano de aula de Sara e Vitória sobre o uso do TinkerPlots ${ }^{\mathrm{TM}}$ no ensino de conceitos estatísticos, as FP apresentam possíveis resoluções da tarefa por parte dos alunos (Figura 5) e indicam que "durante toda a resolução da tarefa, o aluno permanece sentado no computador, trabalha colaborativamente, analisa e interpreta os gráficos. Relaciona os conceitos estatísticos e físicos para fundamentar as possíveis respostas de cada questão" (RT10). 
Possíveis Resoluções:

- Utiliza o gráfico com os atributos: dia do ano por ano.

- Utiliza o gráfico com os atributos: mês por ano.

- Utiliza o gráfico com os atributos: mês por dia do ano.

- Recorre a uma escala contínua ou por classes.

- Calcula as médias da ocorrência do dia do ano separando os anos por classes.

- Calcula a percentagem de ocorrência de cada mês para cada classe do ano.



Figura 5. Resoluções previstas para a tarefa formulada no plano de aula elaborado por Sara e Vitória (RT10).

Ainda sobre a articulação entre conhecimento tecnológico e didático, foi também evidenciado o interesse dos FP por continuar a explorar as ferramentas tecnológicas com o propósito de integrá-las na sua prática profissional. Glória exemplifica-o ao sugerir que "usando os softwares que já conhecemos como o Excel, GeoGebra e outros, podemos deles extrair o que é importante para os objetivos da nossa aula, criando assim uma applet, apenas com conhecimentos básicos de informática e do software" (RT11). Os FP também identificaram as potencialidades de uma ferramenta tecnológica e a sua aplicabilidade no ensino e na aprendizagem da Matemática, como no caso de Sofia que reconheceu "a parte de apresentar o software e perceber as potencialidades de cada um, o que poderíamos fazer com cada um, foi a parte mais importante da nossa aprendizagem" (E).

No geral, em ambos os ciclos, os dados do questionário (Figura 6) revelam que todos os FP reconheceram terem adquirido conhecimentos e desenvolvido capacidades relativas à elaboração de um plano de aula que integre a tecnologia. 
DOI: $10.20396 /$ zet.v29i00.8661780

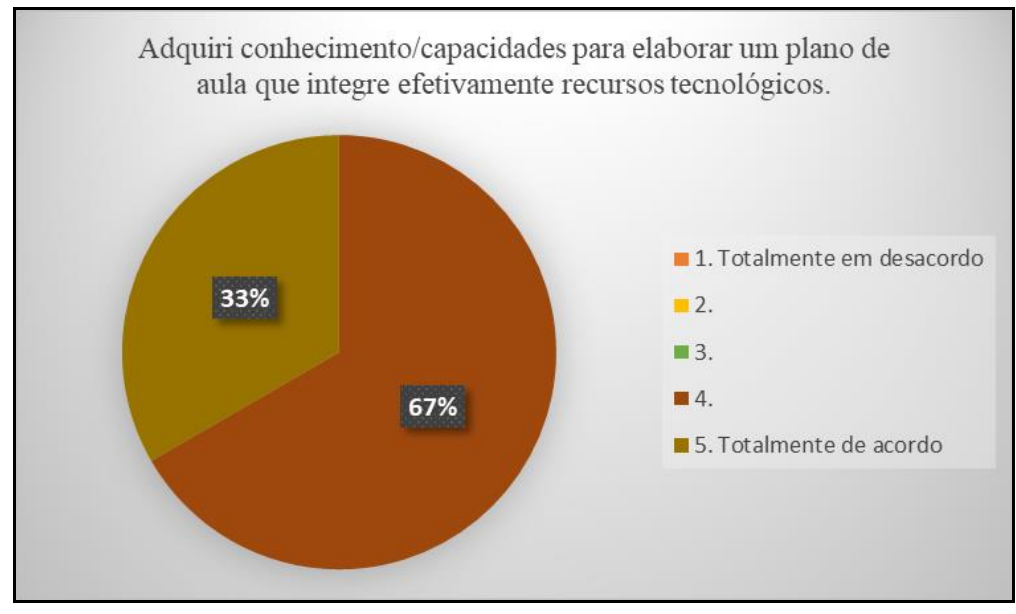

Figura 6. Respostas à questão 8 do Questionário

No que respeita à exploração de ferramentas tecnológicas (P6), os FP destacaram as oportunidades que a experiência de formação lhes ofereceu para usar e explorar diversos recursos tecnológicos disponíveis para o ensino e a aprendizagem da Matemática. Por exemplo, Paula afirmou que "durante o decorrer da disciplina tivemos oportunidade de ter contacto com algumas das tecnologias que podem ser usadas em contexto de sala de aula, nomeadamente o Geogebra e o TinkerPlots ${ }^{\mathrm{TM}}$ " (RT11). Semelhantemente, Sofia referiu que "para nós, como FP de matemática, tem sido importante para a nossa formação o estudo das várias tecnologias atualmente disponíveis" (RT5), e na entrevista salientou algumas destas tecnologias: "quando pensávamos em tecnologias na sala de aula era a projeção de um PowerPoint e quanto muito a calculadora, pelo que temos percebido que há outros, nomeadamente as applets, que não tinha ideia que podíamos usar, e que é um recurso bastante útil" (Sofia, E).

Outro aspeto que os FP reconheceram foi os contributos que a exploração destes recursos lhes trouxe ao seu TK. Glória, por exemplo, referiu que a tarefa sobre a exploração de applets (T7) permitiu-lhe usar "um recurso com que não tinha grande contato e me ajudou a pesquisar mais a fundo o desconhecido. Então fiz dessa tarefa uma oportunidade de conhecer mais uma ferramenta" (RT7). As applets e a plataforma WIX constituíram uma novidade para os FP. Segundo o questionário final (Figura 7), mais da metade dos FP concordaram em terem adquirido conhecimentos relativos ao uso destas ferramentas para o ensino e a aprendizagem da Matemática. 
DOI: $10.20396 /$ zet.v29i00.8661780



Figura 7. Respostas à questão 5 do Questionário

No caso da elaboração de um portfólio digital (T12), os FP concluíram que adquiriram aprendizagens como resultado da exploração e utilização das ferramentas disponíveis na plataforma WIX.com associadas à criação de uma página web. Como Sofia (Figura 8) afirmou, "aprender uma plataforma nova como o WIX e o editor de vídeo como o POWTOON, foram ótimas consequências na elaboração do portefólio” (RT11).



Figura 8. Captura de imagem do vídeo elaborado por Sofia (RT12)

No entanto, os FP também reconheceram que uma das limitações associadas ao uso de várias tecnologias ao longo desta experiência de formação, consistiu no pouco tempo dedicado a aprofundar cada uma delas, evidenciado pela argumentação de Ana: "as tarefas incluíram uma grande diversidade de ferramentas, mas eu gostava ter ido um bocadinho mais a fundo, gostava ter tido tempo para aprofundar, para me familiarizar na ferramenta, e acho que acabou por não acontecer" (E). Este aspeto pode ser considerado uma limitação desta experiência de formação, que pode estar ligada ao próprio contexto no qual decorreu a experiência.

Em relação à participação em espaços de reflexão e partilha de conhecimentos (P7), os resultados revelam que estes espaços, como o portfólio digital, permitiram desenvolver a 
DOI: $10.20396 /$ zet.v29i00.8661780

autorreflexão dos FP sobre a integração da tecnologia na Educação Matemática. No caso da Vitória, esta FP defende que "penso que ainda tenho muitos caminhos a percorrer, mas os contributos da disciplina foram essenciais para esse caminho visto que me levou a poder refletir sobre todos os aspetos que ainda preciso melhorar" (RT11). Já a Sara, especificou alguns dos aspetos submetidos à sua reflexão, indicando que "este foi um trabalho muito enriquecedor, que me levou a refletir sobre aspetos fundamentais, dos quais saliento a elaboração de planos de aula e a utilização de tarefas que integrem a tecnologia" (RT11). Associado a estes resultados, os dados do questionário revelam que todos os FP concordaram terem adquirido capacidades associadas à autorreflexão sobre a integração da tecnologia no ensino e na aprendizagem da Matemática (Figura 9).

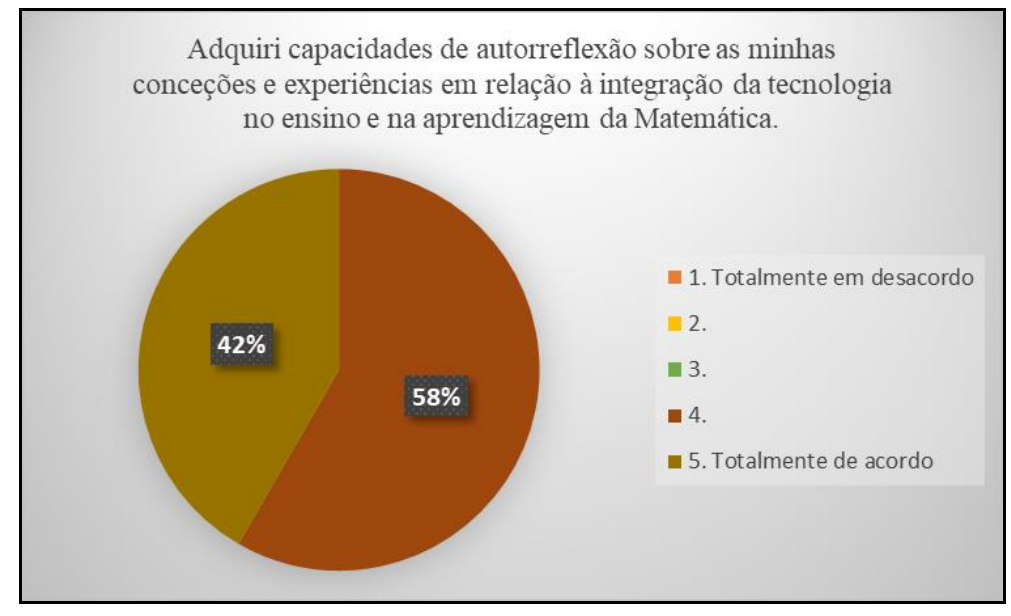

Figura 9. Respostas à questão 1 do Questionário

Em termos gerais, os dados do questionário (Figura 10) também evidenciam que os FP estão de acordo com o facto da experiência de formação ter contribuido para o desenvolvimento do seu conhecimento profissional para integrar efetivamente a tecnologia na Educação Matemática.

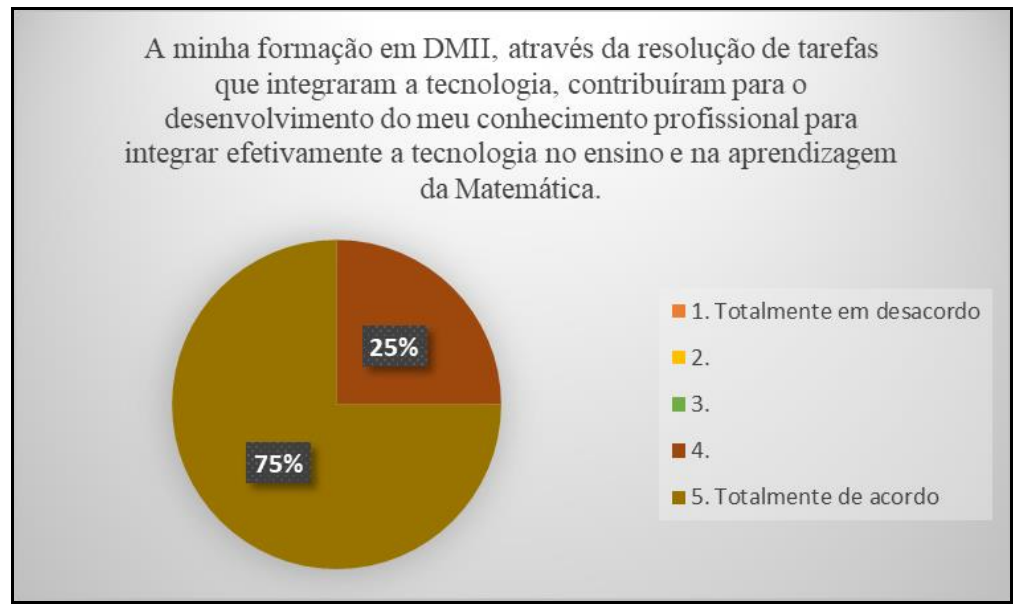

Figura 10. Respostas à questão 9 do Questionário 
DOI: $10.20396 /$ zet.v29i00.8661780

Estes resultados apoiam as opções que orientaram a preparação das tarefas e verificam o cumprimento do objetivo que direcionou a sua implementação na sala de aula desenvolver o TPACK dos FP de Matemática.

\section{Reflexão final}

Este estudo relata os princípios de design de uma experiência de formação inicial que visa desenvolver o TPACK de FP, que é inovadora em Portugal. O reconhecimento dos FP do conhecimento que adquiriram e a análise da operacionalização dos princípios de design durante a experiência ofereceram oportunidade para melhor compreender e confirmar que os princípios de design adotados, que ainda estavam pouco pesquisados, podem contribuir para o promover.

Os resultados obtidos mostram que a experiência de formação baseada no TPACK, realizada neste estudo, contribuiu para os FP de Matemática desenvolverem os conhecimentos TPK e TCK em articulação com o PCK. Em particular, a resolução das tarefas propostas organizadas sequencialmente na Trajetória de Formação e Aprendizagem foram o recurso principal na promoção destes conhecimentos, sendo que a problematização de situações de ensino e aprendizagem da Matemática através de tarefas abertas (Fase 2 e Fase 3) permitiu aos FP explorarem diferentes tecnologias em contextos educativos, contribuindo para desenvolverem o $\mathrm{TK}$, e reconhecer as suas potencialidades articulando esse conhecimento com o TCK e o TPK para os propósitos previstos nas tarefas. Conclui-se, assim, que esta experiência de formação permitiu aos FP desenvolverem conhecimento relativo ao PCK em simultâneo com o TK, o TCK, o TPK e, consequentemente, com o TPACK (Koehler et al., 2014), correspondendo ao que Niess (2012a) defende como o foco dos programas de formação inicial.

Outra conclusão evidente é que esta experiência de formação consolidou as conceções dos FP, estando cada vez mais favoráveis a integrar a tecnologia no ensino e na aprendizagem da Matemática. Tal como noutros estudos (Niess \& Gillow-Wiles, 2017; Niess, 2012b), confirma-se que a mudança nas sua conceções resultou das caraterísticas do programa de formação em que participam. Neste estudo, as caraterísticas da experiência que contribuíram efetivamente para essas mudanças são: (i) ofereceu oportunidade de reflexão, discussão e análise de situações de ensino e aprendizagem da Matemática que integram a tecnologia; (ii) permitiu aos FP explorarem diversas tecnologias para o ensino e aprendizagem da Matemática; e (iii) promoveu a mobilização, articulada, do PCK, TPK e TCK dos FP na planificação de situações de ensino e aprendizagem da Matemática que integrem a tecnologia.

Associado ao conhecimento de diferentes ferramentas tecnológicas, os resultados revelam que os FP desenvolveram e consolidaram significativamente o seu TK. Este conhecimento, segundo Costa et al. (2008), embora fundamental para a compreensão das potencialidades das tecnologias para a aprendizagem, deve ser complementado com oportunidades para os FP as experimentarem "em situações concretas de ensino e 
aprendizagem" (p. 42). Assim, podemos concluir que esta experiência não se limitou a contribuir para desenvolver o TK dos FP, pois também criou oportunidades para que experimentassem esse TK em situações concretas como a resolução de tarefas matemáticas com recurso à tecnologia e na planificação de aulas que integram o uso de ferramentas tecnológicas.

Finalmente, na experiência apresentada neste artigo também se verificou que, durante o processo de formação, os FP reforçaram competências que se aproximam ao segundo nível do framework da UNESCO (2008): o aprofundamento do conhecimento. Por exemplo, os resultados mostram que os FP foram capazes de: ter um pensamento crítico e reflexivo sobre a integração da tecnologia na escola; aumentar o seu interesse e curiosidade sobre o uso de ferramentas tecnológicas para o ensino e a aprendizagem da Matemática; identificar as potencialidades de ferramentas tecnológicas específicas e a sua aplicabilidade na Educação Matemática; e ter uma atitude de perseverança e confiança na integração da tecnologia no ensino e aprendizagem da Matemática.

Como resultado da reflexão feita sobre os resultados obtidos neste estudo e o acompanhamento como formadores das suas fases de experimentação, identificamos que uma limitação da experiência de formação foi não ter sido possível consolidar o conhecimento sobre: a gestão curricular dos recursos tecnológicos em sala de aula, as estratégias de ensino diversificadas para integrar eficientemente a tecnologia, e a adaptação das estratégias de avaliação quando se integra a tecnologia no ensino e na aprendizagem da Matemática. Esta limitação está principalmente relacionada com o contexto da disciplina de Didática onde foi realizada a experiência, na qual não foi possível propor uma tarefa que solicitasse aos FP a implementação de uma aula com recurso à tecnologia com alunos do $7 .^{\circ}$ ao $12 .^{\circ}$ anos por ser uma unidade curricular do $1 .^{\circ}$ ano curso de Mestrado em Ensino de Matemática. Sendo "importante que os FP tenham mais oportunidades de entrar em contato com experiências concretas de uso da tecnologia com os alunos e, desejavelmente, de levarem os seus planos à prática, refletindo sobre a sua implementação em sala de aula" (Oliveira, Henriques \& Gutiérrez-Fallas, 2018, p. 441), uma proposta para ultrapassar esta limitação é, na experiência de formação, oferecer oportunidades para os FP não só planificarem situações de ensino e aprendizagem mas também dinamizarem essas situações nas aulas, como práticas de micro-ensino (Niess \& Gillow-Wiles, 2017), onde podem lecionar uma aula com tecnologia aos seus colegas.

Os resultados deste estudo salientam, assim, principios de design valiosos para a formação inicial de professores, que se confirmam serem promotores do desenvolvimento do TPACK dos futuros professores como o conhecimento profissional necessário para integrar efetivamente a tecnologia no ensino e na aprendizagem da Matemática, aproximando-os da realidade esperada da sua prática futura. 


\section{Referências}

Albuquerque, C., Veloso, C., Rocha, I., Santos, L., Serrazina, L., \& Nápoles, S. (2006). A matemática na formação inicial de professores. Lisboa: APM e Secção de Educação e Matemática da SPCE.

Association of Mathematics Teacher Educators (AMTE) (2017). AMTE Standards for Preparing Teachers of Mathematics. Recuperado de http://www.amte.net/publications.

Canavarro, A. P. (2011). Ensino exploratório da Matemática: Práticas e desafios. Educação e Matemática, 115, 11-17.

Cobb, P., Confrey, J., diSessa, A., Lehere, R., \& Schauble, L. (2003). Design experiments in education research. Educational Researcher, 32(1), 9-13.

Costa, F. (Coord.), Peralta, H., Rodrigues, A., Dias, P., Osório, A. J., Gomes, M. J., Ramos, A., Ramos, J. L., Sebastião, L., Maio, V., \& Valente, L. (2008). Competências TIC. Estudo de Implementação (vol. I). Lisboa: GEPE-Ministério da Educação.

Coutinho, C. P. (2011). Metodologia de Investigação em Ciências Sociais e Humanas, Teoria e Prática. Coimbra: Edições Almedina.

Earle, R. S. (2002). The integration of instructional technology into public education: Promises and challenges. ET Magazine, 42(1), 5-13.

Grossman, P. L. (1989). A study in contrast: Sources of pedagogical content knowledge for secondary English. Journal of Teacher Education, 40(5), 24-31.

Gutiérrez-Fallas, L. F. (2019). O conhecimento tecnológico e pedagógico do conteúdo (TPACK) na formação inicial de professores de matemática do 3. ${ }^{\circ}$ ciclo do ensino básico e do ensino secundário. Tese de Doutoramento, Universidade de Lisboa, Portugal.

Gutiérrez-Fallas, L. F., \& Henriques, A. (2020). O TPACK de futuros professores de Matemática numa Experiência de Formação. Revista Latinoamericana de Investigación en Matemática Educativa, 23(2), 175-202. https://doi.org/10.12802/relime.20.2322

Koehler, M. J., Mishra, P., Kereluik, K., Shin, T. S., \& Graham, C. R. (2014). The technological pedagogical content knowledge framework. In J. Spector, M. Merrill, J. Elen, \& M. Bishop (Eds.), Handbook of research on educational communications and technology (pp. 101-111). New York, NY: Springer.

Krumsvik, R. J. (2014). Teacher educators' digital competence. Scandinavian Journal of Educational Research, 58(3), 269-280.

Leung, A. (2017). Exploring techno-pedagogic task design in the mathematics classroom. In A. Leung \& A. Baccaglini-Frank (Eds.), Digital technologies in designing mathematics education tasks: Potential and pitfalls (pp. 3-16). Cham: Springer.

Mishra, P., \& Koehler, M. J. (2006). Technological pedagogical content knowledge: A framework for integrating technology in teachers' knowledge. Teachers College Record, 108(6), 1017-1054.

National Council of Teachers of Mathematics (NCTM) (2014). Principles to actions: Ensuring mathematical success for all. Reston, VA: Author. 
Niess, M., \& Gillow-Wiles, H. (2017). Expanding teachers' technological pedagogical reasoning with a systems pedagogical approach. Australasian Journal of Educational Technology, 33(3), 77-95.

Niess, M. L. (2012a). Rethinking pre-service mathematics teachers' preparation: technological, pedagogical and content knowledge (TPACK). In D. Polly, C. Mims and K. Persichitte (Eds.), Developing technology-rich, teacher education programs: Key issues (pp. 316-336). Hershey, PA: IGI Global.

Niess, M. L. (2012b). Teacher Knowledge for Teaching with Technology: A TPACK lens. In R. Ronau, C. Rakes and M. Niess (Eds.), Educational technology, teacher knowledge, and classroom impact: A research handbook on frameworks and approaches (pp. 1-15). Hershey, PA: IGI Global.

Oliveira, H., Henriques, A., \& Gutiérrez-Fallas, L. F. (2018). A integração da tecnologia na planificação de aulas na perspectiva do ensino exploratório: um estudo com futuros professores de Matemática. PERSPECTIVA, 36(2), 421-446.

Ponte, J. P., \& Chapman, O. (2008). Preservice mathematics teachers' knowledge and development. In L. English (Ed.), Handbook of international research in mathematics education ( $2^{\text {nd }}$ ed., pp. 225-263). New York, NY: Routledge.

Ponte, J. P. (2012). Estudiando el conocimiento y el desarrollo profesional del profesorado de matemáticas. Teoría, crítica y práctica de la educación matemática, 41, 83-98.

Ponte, J. P., Carvalho, R., Mata-Pereira, J., \& Quaresma, M. (2016). Investigação baseada em design para compreender e melhorar as práticas educativas. Quadrante, 25(2), 77-98.

Shulman, L. S. (1986). Those who understand: Knowledge growth in teaching. Educational Researcher, 15(2), 4-14.

UNESCO (2008). ICT Competency Standards for Teachers. Policy Framework. Paris: UNESCO. 